\title{
Prevalence of Urinary Tract Infection in Nephrotic Syndrome at Zagazig University Children Hospital
}

\author{
Ali Mohammed Abo Zeid ${ }^{1}$, Mayy Abdel-Fattah Mohammed ${ }^{1}$, Rehab Hosny El-Sokary², \\ Hosny Hussien Hosny Ahmed*1
}

Departments of ${ }^{1}$ Pediatrics and ${ }^{2}$ Microbiology and Immunology, Faculty of Medicine, Zagazig University

*Corresponding author: Hosny Hussien Hosny Ahmed, Mobile: (+20) 01006714949, E-Mail: dr.hosnyaser@gmail.com

\begin{abstract}
Background: Nephrotic syndrome (NS) is the most common chronic glomerular diseases in children. Nephrotic syndrome increases a child's susceptibility to infection. Urinary tract infection (UTI) is the second most common bacterial disease in children after upper respiratory tract infection.

Aim of this work: To find out the prevalence of UTI (either community acquired or hospital acquired) in NS children and to uncover the bacterial spectrum and their sensitivity pattern for selecting the empirical antibiotic therapy till the results of culture are awaited.

Subjects and methods: This study was descriptive cross sectional. 33 nephrotic syndrome cases were included in study. All patients were subjected to full history taking, complete clinical examination and investigations as urine analysis and urine culture. Results: The percentage of community acquired urinary tract infection (CA-UTI) was $57.6 \%$ and hospital acquired urinary tract infection (HA-UTI) was $42.4 \%$. E. coli is the commonest organism causing UTI followed by klebsiella and proteus mirabilis. The isolated organisms showed resistance to many oral and parenteral antibiotics. Amikacin gentamycin may be the first option of empiric therapy while waiting for culture reports. Conclusion: Children with nephrotic syndrome are frequently predisposed to UTI and in most cases it is asymptomatic often undiagnosed. Identifying bacterial causes and their susceptibility pattern of UTI will help in selecting the empirical antibiotic therapy till the results of culture are awaited. Although dipstick and microscopic urinalysis can support the diagnosis of UTI, growth of a single type of bacteria on urine culture is the gold standard.
\end{abstract}

Key words: Urinary Tract Infection- Nephrotic Syndrome- Prevalence.

\section{INTRODUCTION}

Nephrotic syndrome is most prevalent in children with chronic glomerular diseases. In children the incidence of this syndrome is $2-7$ cases per $100.0000^{(\mathbf{1})}$. Most (90 per cent) cases of nephrotic syndrome are idiopathic. The other $10 \%$ have a secondary idiopathic nephrotic syndrome to glomerular or systemic diseases (2). Nephrotic syndrome patients suffer from various complications such as infection, effusion, hypovolemia, hyperlipidemia and hypercoagulability (3). When children suffering from nephrotic syndrome reach the pediatric ward in the hospital, it is essential to examine them first in order to avoid possible complications or immediate discovery of infections and to initiate appropriate therapy ${ }^{(\mathbf{4})}$.

Nephrotic syndrome makes the child more susceptible to infection. Urinary tract infection is the 2nd most common bacterial disease in children following infection of the upper respiratory tract and is the most prevalent bacterial infection that affects humans throughout their lives ${ }^{(5)}$.

Symptoms of the urinary tract infection differ in age and the younger the child the much more nonspecific these symptoms are ${ }^{(6)}$.
The aim of this work was to find out the prevalence of UTI (either community acquired or hospital acquired) in NS children and to uncover the bacterial spectrum and their sensitivity pattern for selecting the empirical antibiotic therapy till the results of culture are awaited.

\section{SUBJECTS AND METHODS}

\section{Technical design:}

Study design: A descriptive cross-sectional study, carried out at a Pediatric Nephrology Unit of Children Hospital and Medical Microbiology and Immunology Department, Faculty of Medicine, Zagazig University. It was carried out over a period of twelve months from February 2018 to January 2019.

Subjects: Thirty-three nephrotic syndrome cases were included in study. This study was designed to assess the prevalence of urinary tract infections (either community acquired or hospital acquired) and bacterial spectrum and their sensitivity pattern in children with nephrotic syndrome either newly diagnosed (1st attack) or relapsed cases of nephrotic syndrome attending at children hospital. 
Ethical approval:

The study was approved by the Ethics Board of Zagazig University and an informed written consent was taken from each participant in the study.

\section{Case definition:}

- HA-UTI is a urinary tract infected patient that was not manifested or incubated at the time of admission. Infection may appear even after discharge (7).

- CA-UTI has been defined as any urinary tract infection that tends to occur in the community, or as the second most commonly diagnosed infection in the community in less than 48 hours of hospital admission (7).

Inclusion criteria: Children from 6 months up to 15 years, both male and female children and cases of nephrotic syndrome either newly diagnosed or relapsed were included.

Exclusion criteria: Children less than 6 months, above 15 years, who were already on antibiotics either oral or parenteral in the last 48 hours or with urogenital malformations.

\section{Methods:}

All patients were subjected to the following:

A- Full history taking with special focus on the presence of urological manifestations (dysuria, loin pain, frequency, supra-pubic pain, change of the color of urine, offensive smell of urine, enuresis), treatment with certain drugs for long period and past history of renal diseases or family history of renal diseases.
B-Complete clinical examination with special focus on weight, edema and abdominal examination (for renal mass or fullness of renal angles).

C- Investigations:

1- Urine analysis: Urine samples from children were obtained then complete urine analysis was done on this urine specimens by microscopic urine examination and dipstick analysis.

2- Urine cultures were done.

The pattern of antibiotic susceptibility was done by the method of diffusion of the disks.

- Detection of most prevalent multi drug resistant (MDR) pathogen: MDR was defined as nonsusceptibility to at least one agent in three or more antimicrobial categories. (8)

Administrative considerations: Written informed consent was obtained from all participants or their parents and the study was approved by the Research Ethical Committee of Faculty of Medicine, Zagazig University (Institutional Research Board IRB). The work was carried out in accordance with World Medical Association's Code of Ethics (Helsinki Declaration) for human involvement studies.

\section{Data management and Statistical analysis:}

The data were coded, entered and processed on computer using statistical package for social science (SPSS) version 18. The results were presented then interpreted in tabular and diagrammatic forms. Mean, as descriptive figures; standard deviation, frequency, range and percentage are used.

\section{RESULTS}

Table (1): Frequency of UTI of the study population

\begin{tabular}{|l|c|c|}
\hline \multicolumn{1}{|c|}{ Types of UTI } & \multicolumn{2}{|c|}{ UTI patients (n=33) } \\
\cline { 2 - 3 } & $\mathbf{N}$ & $\mathbf{\%}$ \\
\hline Community Acquired & 19 & $57.6 \%$ \\
\hline H. Acquired & 14 & $42.4 \%$ \\
\hline
\end{tabular}

Table (1) showed that the percentage of CA-UTI was $57.6 \%$ and HA-UTI was $42.4 \%$.

Table (2): Comparison between CA- UTI and HA- UTI regarding organism

\begin{tabular}{|l|c|c|c|c|}
\hline \multirow{2}{*}{ Organism } & \multicolumn{2}{|c|}{ CA- UTI (n=19) } & \multicolumn{2}{c|}{ HA- UTI (n=14) } \\
\cline { 2 - 5 } & $\mathbf{N}$ & $\mathbf{\%}$ & $\mathbf{N}$ & $\mathbf{\%}$ \\
\hline E. coli & 6 & $31.6 \%$ & 8 & $57.1 \%$ \\
\hline Klebsiella pneumonia & 4 & $21.1 \%$ & 2 & $14.3 \%$ \\
\hline Proteus mirabilis & 4 & $21.1 \%$ & 2 & $14.3 \%$ \\
\hline Staph aureus & 4 & $21.1 \%$ & 1 & $14.3 \%$ \\
\hline Enterococcus faecalis & 1 & $5.3 \%$ & 1 & $7.1 \%$ \\
\hline
\end{tabular}

Table (2) showed that the highest percentage among the organisms was E. coli (57.1\% \& $31.6 \%)$ in HA-UTI and CA- UTI respectively. Klebsiella and Proteus were higher in CA-UTI than HA-UTI $(21.1 \%$ \& $14.3 \%$ respectively). The least percentage was Staph and Enterococcus faecalis. 
Table (3): Multidrug resistant (MDR) distribution according to UTI types

\begin{tabular}{|c|c|c|}
\hline \multirow{2}{*}{ MDR } & \multicolumn{2}{|c|}{ UTI patients $(\mathbf{n}=33)$} \\
\cline { 2 - 3 } & CA- UTI & HA-UTI \\
\hline
\end{tabular}

Table (3) demonstrated that antibiotic susceptibility showed that 21 cases (63.6\%) were of MDR type divided into 15 cases $(78.9 \%)$ of community origin and 6 cases $(42.9 \%)$ of hospital acquired origin.

Table (4): Antibiotic susceptibility pattern of Gram-negative bacteria from CA-UTI

\begin{tabular}{|c|c|c|c|c|c|c|c|c|c|c|}
\hline & & & & am-neg & ive bac & & & & & \\
\hline Antibiotics & & Esch & ichia col & $(n=6)$ & & bsiella (r & & & oteus $(n=$ & \\
\hline & & I & $\mathrm{R}$ & $\mathrm{S}$ & I & $\mathrm{R}$ & $\mathrm{S}$ & I & $\mathrm{R}$ & $S$ \\
\hline Ampicillin & No. & 0 & 6 & 0 & 0 & 4 & 0 & 0 & 4 & 0 \\
\hline & $\%$ & $00.0 \%$ & $100 \%$ & $00.0 \%$ & $0.0 \%$ & $100.0 \%$ & $0.0 \%$ & $0.0 \%$ & $100.0 \%$ & $0.0 \%$ \\
\hline Ciprofloxacin & No. & 1 & 0 & 5 & 2 & 1 & 1 & 1 & 1 & 2 \\
\hline & $\%$ & $16.7 \%$ & $0.0 \%$ & $83.3 \%$ & $50.0 \%$ & $25.0 \%$ & $25.0 \%$ & $25.0 \%$ & $25.0 \%$ & $50.0 \%$ \\
\hline Gentamycin & No. & 1 & 0 & 5 & 3 & 0 & 1 & 2 & 0 & 2 \\
\hline & $\%$ & $16.7 \%$ & $0.0 \%$ & $83.3 \%$ & $75.0 \%$ & $0.0 \%$ & $25.0 \%$ & $50.0 \%$ & $0.0 \%$ & $50.0 \%$ \\
\hline Amikacine & No. & 3 & 0 & 3 & 1 & 0 & 3 & 2 & 0 & 2 \\
\hline & $\%$ & $50.0 \%$ & $0.0 \%$ & $50.0 \%$ & $25.0 \%$ & $0.0 \%$ & $75.0 \%$ & $50.0 \%$ & $0.0 \%$ & $50.0 \%$ \\
\hline Nitrofurantoin & No. & 5 & 1 & 0 & 0 & 3 & 1 & 0 & 4 & 0 \\
\hline & $\%$ & $83.3 \%$ & $16.7 \%$ & $0.0 \%$ & $0.0 \%$ & $75.0 \%$ & $25.0 \%$ & $0.0 \%$ & $100.0 \%$ & $0.0 \%$ \\
\hline Sulfamethoxazole & No. & 3 & 1 & 2 & 0 & 4 & 0 & 0 & 4 & 0 \\
\hline & $\%$ & $50.0 \%$ & $16.7 \%$ & $33.3 \%$ & $0.0 \%$ & $100.0 \%$ & $0.0 \%$ & $0.0 \%$ & $100.0 \%$ & $0.0 \%$ \\
\hline Ceftazidime & No. & 3 & 3 & 0 & 2 & 1 & 1 & 2 & 2 & 0 \\
\hline & $\%$ & $50.0 \%$ & $50.0 \%$ & $0.0 \%$ & $50.0 \%$ & $25.0 \%$ & $25.0 \%$ & $50.0 \%$ & $50.0 \%$ & $0.0 \%$ \\
\hline Amoxicillin & No. & 0 & 6 & 0 & 0 & 4 & 0 & 0 & 4 & 0 \\
\hline & $\%$ & $0.0 \%$ & $100.0 \%$ & $0.0 \%$ & $0.0 \%$ & $100.0 \%$ & $0.0 \%$ & $0.0 \%$ & $100.0 \%$ & $0.0 \%$ \\
\hline Ceftriaxone & No. & 2 & 2 & 2 & 2 & 1 & 1 & 2 & 0 & 2 \\
\hline & $\%$ & $33.3 \%$ & $33.3 \%$ & $33.3 \%$ & $50.0 \%$ & $25.0 \%$ & $25.0 \%$ & $50.0 \%$ & $0.0 \%$ & $50.0 \%$ \\
\hline Cefepime & No. & 3 & 3 & 0 & 0 & 4 & 0 & 1 & 0 & 3 \\
\hline & $\%$ & $50.0 \%$ & $50.0 \%$ & $0.0 \%$ & $0.0 \%$ & $100.0 \%$ & $0.0 \%$ & $25.0 \%$ & $0.0 \%$ & $75.0 \%$ \\
\hline Cefoxitine & No. & 1 & 5 & 0 & 1 & 3 & 0 & 0 & 4 & 0 \\
\hline & $\%$ & $16.7 \%$ & $83.3 \%$ & $0.0 \%$ & $25.0 \%$ & $75.0 \%$ & $0.0 \%$ & $0.0 \%$ & $100.0 \%$ & $0.0 \%$ \\
\hline Cefazoline & No. & 1 & 5 & 0 & 1 & 3 & 0 & 0 & 4 & 0 \\
\hline & $\%$ & $16.7 \%$ & $83.3 \%$ & $0.0 \%$ & $25.0 \%$ & $75.0 \%$ & $0.0 \%$ & $0.0 \%$ & $100.0 \%$ & $0.0 \%$ \\
\hline Meronem & No. & 1 & 2 & 3 & 0 & 2 & 2 & 0 & 4 & 0 \\
\hline & $\%$ & $16.7 \%$ & $33.3 \%$ & $50.0 \%$ & $0.0 \%$ & $50.0 \%$ & $50.0 \%$ & $0.0 \%$ & $100.0 \%$ & $0.0 \%$ \\
\hline
\end{tabular}

Table (4) showed the antibiotic susceptibility pattern of Gram-negative bacteria from CA-UTI. 
https://ejhm.journals.ekb.eg/

Table (5): Antibiotic susceptibility pattern of Gram-negative bacteria from HA-UTI

\begin{tabular}{|c|c|c|c|c|c|c|c|c|c|c|}
\hline \multicolumn{11}{|c|}{ Gram-negative bacilli } \\
\hline \multicolumn{2}{|l|}{ Antibiotics } & \multicolumn{3}{|c|}{ Escherichia coli $(n=8)$} & \multicolumn{3}{|c|}{ Klebsiella $(n=2)$} & \multicolumn{3}{|c|}{ Proteus $(n=2)$} \\
\hline & & I & $\mathbf{R}$ & $\mathbf{S}$ & I & $\mathbf{R}$ & $\mathbf{S}$ & I & $\mathbf{R}$ & $\mathbf{S}$ \\
\hline \multirow[t]{2}{*}{ Ampicillin } & No. & 0 & 8 & 0 & 0 & 2 & 0 & 0 & 2 & 0 \\
\hline & $\%$ & $0.0 \%$ & $100.0 \%$ & $0.0 \%$ & $0.0 \%$ & $100.0 \%$ & $0.0 \%$ & $0.0 \%$ & $100.0 \%$ & $0.0 \%$ \\
\hline \multirow[t]{2}{*}{ Ciprofloxacin } & No. & 4 & 0 & 4 & 2 & 0 & 0 & 0 & 1 & 1 \\
\hline & $\%$ & $50.0 \%$ & $0.0 \%$ & $50.0 \%$ & $100.0 \%$ & $0.0 \%$ & $0.0 \%$ & $0.0 \%$ & $50.0 \%$ & $50.0 \%$ \\
\hline \multirow[t]{2}{*}{ Gentamycin } & No. & 2 & 0 & 6 & 1 & 0 & 1 & 1 & 0 & 1 \\
\hline & $\%$ & $25.0 \%$ & $0.0 \%$ & $75.0 \%$ & $50.0 \%$ & $0.0 \%$ & $50.0 \%$ & $50.0 \%$ & $0.0 \%$ & $50.0 \%$ \\
\hline \multirow[t]{2}{*}{ Amikacine } & No. & 4 & 0 & 4 & 0 & 0 & 2 & 1 & 0 & 1 \\
\hline & $\%$ & $50.0 \%$ & $0.0 \%$ & $50.0 \%$ & $0.0 \%$ & $0.0 \%$ & $100.0 \%$ & $50.0 \%$ & $0.0 \%$ & $50.0 \%$ \\
\hline \multirow[t]{2}{*}{ Nitrofurantoin } & No. & 5 & 0 & 3 & 1 & 1 & 0 & 0 & 2 & 0 \\
\hline & $\%$ & $62.5 \%$ & $0.0 \%$ & $37.5 \%$ & $50.0 \%$ & $50.0 \%$ & $0.0 \%$ & $0.0 \%$ & $100.0 \%$ & $0.0 \%$ \\
\hline \multirow[t]{2}{*}{ Sulfamethoxazole } & No. & 5 & 1 & 2 & 0 & 2 & 0 & 0 & 2 & 0 \\
\hline & $\%$ & $62.5 \%$ & $12.5 \%$ & $25.0 \%$ & $0.0 \%$ & $100.0 \%$ & $0.0 \%$ & $0.0 \%$ & $100.0 \%$ & $0.0 \%$ \\
\hline \multirow[t]{2}{*}{ Ceftazidime } & No. & 2 & 6 & 0 & 0 & 0 & 2 & 0 & 2 & 0 \\
\hline & $\%$ & $25.0 \%$ & $75.0 \%$ & $0.0 \%$ & $0.0 \%$ & $0.0 \%$ & $100.0 \%$ & $0.0 \%$ & $100.0 \%$ & $0.0 \%$ \\
\hline \multirow[t]{2}{*}{ Amoxicillin } & No. & 0 & 8 & 0 & 0 & 2 & 0 & 0 & 2 & 0 \\
\hline & $\%$ & $0.0 \%$ & $100.0 \%$ & $0.0 \%$ & $0.0 \%$ & $100.0 \%$ & $0.0 \%$ & $0.0 \%$ & $100.0 \%$ & $0.0 \%$ \\
\hline \multirow[t]{2}{*}{ Ceftriaxone } & No. & 3 & 3 & 2 & 1 & 1 & 0 & 1 & 0 & 1 \\
\hline & $\%$ & $37.5 \%$ & $37.5 \%$ & $25.0 \%$ & $50.0 \%$ & $50.0 \%$ & $0.0 \%$ & $50.0 \%$ & $0.0 \%$ & $50.0 \%$ \\
\hline \multirow[t]{2}{*}{ Cefepime } & No. & 1 & 6 & 1 & 0 & 2 & 0 & 2 & 0 & 0 \\
\hline & $\%$ & $12.5 \%$ & $75.0 \%$ & $12.5 \%$ & $0.0 \%$ & $100.0 \%$ & $0.0 \%$ & $100.0 \%$ & $0.0 \%$ & $0.0 \%$ \\
\hline \multirow[t]{2}{*}{ Cefoxitine } & No. & 2 & 6 & 0 & 0 & 2 & 0 & 0 & 2 & 0 \\
\hline & $\%$ & $25.0 \%$ & $75.0 \%$ & $0.0 \%$ & $0.0 \%$ & $100.0 \%$ & $0.0 \%$ & $0.0 \%$ & $100.0 \%$ & $0.0 \%$ \\
\hline \multirow[t]{2}{*}{ Cefazoline } & No. & 0 & 8 & 0 & 0 & 2 & 0 & 0 & 2 & 0 \\
\hline & $\%$ & $0.0 \%$ & $100.0 \%$ & $0.0 \%$ & $0.0 \%$ & $100.0 \%$ & $0.0 \%$ & $0.0 \%$ & $100.0 \%$ & $0.0 \%$ \\
\hline \multirow[t]{2}{*}{ Meronem } & No. & 0 & 5 & 3 & 1 & 1 & 0 & 0 & 2 & 0 \\
\hline & $\%$ & $0.0 \%$ & $62.5 \%$ & $37.5 \%$ & $50.0 \%$ & $50.0 \%$ & $0.0 \%$ & $0.0 \%$ & $100.0 \%$ & $0.0 \%$ \\
\hline
\end{tabular}

Table (5) showed the antibiotic susceptibility pattern of Gram-negative bacteria from HA-UTI. 
Table (6): Antibiotic susceptibility pattern of Gram- positive bacteria from CA-UTI

\begin{tabular}{|c|c|c|c|c|c|c|c|}
\hline \multicolumn{8}{|c|}{ Gram-positive cocci } \\
\hline \multicolumn{2}{|l|}{ Antibiotics } & \multicolumn{3}{|c|}{ Enterococcus $(n=1)$} & \multicolumn{3}{|c|}{ Staph $(n=4)$} \\
\hline & & I & $\mathbf{R}$ & $\mathbf{S}$ & I & $\mathbf{R}$ & $\mathbf{S}$ \\
\hline \multirow[t]{2}{*}{ Ampicillin } & No. & 0 & 1 & 0 & 1 & 1 & 2 \\
\hline & $\%$ & $0.0 \%$ & $100.0 \%$ & $0.0 \%$ & $25.0 \%$ & $25.0 \%$ & $50.0 \%$ \\
\hline \multirow[t]{2}{*}{ Ciprofloxacin } & No. & 0 & 1 & 0 & 0 & 4 & 0 \\
\hline & $\%$ & $0.0 \%$ & $100.0 \%$ & $0.0 \%$ & $0.0 \%$ & $100.0 \%$ & $0.0 \%$ \\
\hline \multirow[t]{2}{*}{ Gentamycin } & No. & 0 & 1 & 0 & 0 & 4 & 0 \\
\hline & $\%$ & $0.0 \%$ & $100.0 \%$ & $0.0 \%$ & $0.0 \%$ & $100.0 \%$ & $0.0 \%$ \\
\hline \multirow[t]{2}{*}{ Nitrofurantoin } & No. & 0 & 0 & 1 & 1 & 1 & 2 \\
\hline & $\%$ & $0.0 \%$ & $0.0 \%$ & $100.0 \%$ & $25.0 \%$ & $25.0 \%$ & $50.0 \%$ \\
\hline \multirow[t]{2}{*}{ Sulfamethoxazole } & No. & 1 & 0 & 0 & 0 & 3 & 1 \\
\hline & $\%$ & $100.0 \%$ & $0.0 \%$ & $0.0 \%$ & $0.0 \%$ & $75.0 \%$ & $25.0 \%$ \\
\hline \multirow[t]{2}{*}{ Cefoxitine } & No. & 0 & 1 & 0 & 0 & 4 & 0 \\
\hline & $\%$ & $0.0 \%$ & $100.0 \%$ & $0.0 \%$ & $0.0 \%$ & $100.0 \%$ & $0.0 \%$ \\
\hline \multirow[t]{2}{*}{ Vancomycin } & No. & 0 & 1 & 0 & 1 & 2 & 1 \\
\hline & $\%$ & $0.0 \%$ & $100.0 \%$ & $0.0 \%$ & $25.0 \%$ & $50.0 \%$ & $25.0 \%$ \\
\hline \multirow[t]{2}{*}{ Erthromycin } & No. & 0 & 1 & 0 & 2 & 0 & 2 \\
\hline & $\%$ & $0.0 \%$ & $100.0 \%$ & $0.0 \%$ & $50.0 \%$ & $0.0 \%$ & $50.0 \%$ \\
\hline \multirow[t]{2}{*}{ Clindamycin } & No. & 0 & 1 & 0 & 0 & 4 & 0 \\
\hline & $\%$ & $0.0 \%$ & $100.0 \%$ & $0.0 \%$ & $0.0 \%$ & $100.0 \%$ & $0.0 \%$ \\
\hline \multirow[t]{2}{*}{ Linezolide } & No. & 1 & 0 & 0 & 0 & 3 & 1 \\
\hline & $\%$ & $100.0 \%$ & $0.0 \%$ & $0.0 \%$ & $0.0 \%$ & $75.0 \%$ & $25.0 \%$ \\
\hline \multirow[t]{2}{*}{ Rifampicin } & No. & 0 & 1 & 0 & 0 & 2 & 2 \\
\hline & $\%$ & $0.0 \%$ & $100.0 \%$ & $0.0 \%$ & $0.0 \%$ & $50.0 \%$ & $50.0 \%$ \\
\hline \multirow[t]{2}{*}{ Amxocilline-Cluavulenic acid } & No. & 0 & 1 & 0 & 1 & 1 & 2 \\
\hline & $\%$ & $0.0 \%$ & $100.0 \%$ & $0.0 \%$ & $25.0 \%$ & $25.0 \%$ & $50.0 \%$ \\
\hline
\end{tabular}

Table (6) showed the antibiotic susceptibility pattern of Gram-positive bacteria from CA-UTI.

Table (7): Antibiotic susceptibility pattern of Gram- positive e bacteria from HA-UTI

\begin{tabular}{|c|c|c|c|c|c|c|c|}
\hline \multicolumn{8}{|c|}{ Gram-positive cocci } \\
\hline \multicolumn{2}{|c|}{ Antibiotics } & \multicolumn{3}{|c|}{ Enterococcus $(n=1)$} & \multicolumn{3}{|c|}{ Staph $(n=1)$} \\
\hline & & I & $\mathbf{R}$ & $\mathbf{S}$ & I & $\mathbf{R}$ & $\mathbf{S}$ \\
\hline \multirow[t]{2}{*}{ Ampicillin } & No. & 0 & 1 & 0 & 0 & 1 & 0 \\
\hline & $\%$ & $0.0 \%$ & $100.0 \%$ & $0.0 \%$ & $0.0 \%$ & $100.0 \%$ & $0.0 \%$ \\
\hline \multirow[t]{2}{*}{ Ciprofloxacin } & No. & 0 & 1 & 0 & 0 & 1 & 0 \\
\hline & $\%$ & $0.0 \%$ & $100.0 \%$ & $0.0 \%$ & $0.0 \%$ & $100.0 \%$ & $0.0 \%$ \\
\hline \multirow[t]{2}{*}{ Gentamycin } & No. & 0 & 1 & 0 & 0 & 1 & 0 \\
\hline & $\%$ & $0.0 \%$ & $100.0 \%$ & $0.0 \%$ & $0.0 \%$ & $100.0 \%$ & $0.0 \%$ \\
\hline \multirow{2}{*}{ Nitrofurantoin } & No. & 0 & 1 & 0 & 1 & 0 & 0 \\
\hline & $\%$ & $0.0 \%$ & $100.0 \%$ & $0.0 \%$ & $100.0 \%$ & $0.0 \%$ & $0.0 \%$ \\
\hline \multirow[t]{2}{*}{ Sulfamethoxazole } & No. & 0 & 1 & 0 & 0 & 1 & 0 \\
\hline & $\%$ & $0.0 \%$ & $100.0 \%$ & $0.0 \%$ & $0.0 \%$ & $100.0 \%$ & $0.0 \%$ \\
\hline \multirow[t]{2}{*}{ Cefoxitine } & No. & 0 & 1 & 0 & 0 & 1 & 0 \\
\hline & $\%$ & $0.0 \%$ & $100.0 \%$ & $0.0 \%$ & $0.0 \%$ & $100.0 \%$ & $0.0 \%$ \\
\hline \multirow[t]{2}{*}{ Vancomycin } & No. & 0 & 1 & 0 & 0 & 0 & 1 \\
\hline & $\%$ & $0.0 \%$ & $100.0 \%$ & $0.0 \%$ & $0.0 \%$ & $0.0 \%$ & $100.0 \%$ \\
\hline \multirow[t]{2}{*}{ Erythromycin } & No. & 0 & 1 & 0 & 1 & 0 & 0 \\
\hline & $\%$ & $0.0 \%$ & $100.0 \%$ & $0.0 \%$ & $100.0 \%$ & $0.0 \%$ & $0.0 \%$ \\
\hline \multirow[t]{2}{*}{ Clindamycin } & No. & 0 & 1 & 0 & 0 & 0 & 1 \\
\hline & $\%$ & $0.0 \%$ & $100.0 \%$ & $0.0 \%$ & $0.0 \%$ & $0.0 \%$ & $100.0 \%$ \\
\hline \multirow[t]{2}{*}{ Linezolide } & No. & 0 & 1 & 0 & 0 & 0 & 1 \\
\hline & $\%$ & $0.0 \%$ & $100.0 \%$ & $0.0 \%$ & $0.0 \%$ & $0.0 \%$ & $100.0 \%$ \\
\hline \multirow[t]{2}{*}{ Rifampicin } & No. & 0 & 1 & 0 & 0 & 1 & 0 \\
\hline & $\%$ & $0.0 \%$ & $100.0 \%$ & $0.0 \%$ & $0.0 \%$ & $100.0 \%$ & $0.0 \%$ \\
\hline \multirow[t]{2}{*}{ Amxocilline-Clavulenic acid } & No. & 0 & 1 & 0 & 0 & 1 & 0 \\
\hline & $\%$ & $0.0 \%$ & $100.0 \%$ & $0.0 \%$ & $0.0 \%$ & $100.0 \%$ & $0.0 \%$ \\
\hline
\end{tabular}

Table (7) showed the antibiotic susceptibility pattern of Gram-positive bacteria from HA-UTI. 


\section{DISCUSSION}

Nephrotic syndrome is a common renal disorder in pediatric population, which predisposes to various systemic infections. Immunosuppression may mask the typical clinical presentation of infections in NS and proper management might be delayed. Understanding the pattern of infections is important in the appropriate management of these children and to institute preventive measures ${ }^{(9)}$. Of all infections, urinary tract infection is of special interest because most of the urinary tract infection in nephrotic syndrome are asymptomatic and can be easily missed if active search is not made. Besides, in general the younger the child the less localized the signs and symptoms of urinary tract infection are ${ }^{(\mathbf{1 0})}$.

The present study was conducted to find out the prevalence of UTI (either community acquired or hospital acquired) in NS children and to uncover the bacterial spectrum and their sensitivity pattern for selecting the empirical antibiotic therapy till the results of culture are awaited.

Infections of the urinary tract remain commonly diagnosed in outpatients and hospitalized patients. Our study showed that the most common organisms causing CA-UTI in NS patients were Escherichia coli $(31.6 \%)$, followed by Proteus mirabilis as well as Klebsiella pneumonia and Staphylococcus aureus (21.1\% each). The least common organisms in the study causing CA-UTI in NS was Enterococcus faecalis $(5.3 \%)$. These results agree with Nimri et al. (11) who published that CA-UTI is generally caused by Escherichia coli and other members of the family Enterobacteriaceae.

The current study found that the most common organisms causing HA-UTI in NS patients were Escherichia coli (57.1\%), followed by Proteus mirabilis as well as Klebsiella pneumonia (14.3\% each), while the least common organisms in the study causing HA-UTI in NS were Enterococcus faecalis and Staphylococcus aureus. Finally, the most common organisms causing both CA-UTI and HAUTI in NS patients were Escherichia coli but more in HA-UTI, followed by Proteus mirabilis as well as Klebsiella pneumonia but more in CA-UTI. Our results are supported by Edlin et al. ${ }^{(12)}$ and Paschke et al. ${ }^{(13)}$.

Current study showed that multidrug resistant to CA-UTI was in 15 cases $(78.9 \%)$ and to HA-UTI was in 6 cases $(42.9 \%)$. Our results worried about and reflected that the overuse of antibiotics had increased extensively these days and this intense expansion in use of antibiotics had significantly intensified the antibiotic resistance. As with any infection treatment, the function of antibiotics is very crucial in UTI. The resistant forms have greater frequency variation with regard to localities and age groups. In many researches the organisms that cause UTI have shown multidrug resistance. In addition, Ronald ${ }^{(14)}$ found that pathogens traditionally related to UTI change many of their characteristics, especially due to antimicrobial resistance.

Antibiotic sensitivity tests revealed that the most common antibiotics to which Escherichia coli were sensitive were gentamycin $(78.6 \%)$ followed by ciprofloxacin $(64.3 \%)$ followed by amikacin $(50 \%)$ then meropenem $(42.6 \%)$. The remaining cultures were sensitive to ceftriaxone $(35.7 \%)$, sulfamethoxazole $(28.6 \%)$, nitrofurantoin $(21.4 \%)$ and cefepime $(7.1 \%)$. Mazzalli ${ }^{(15)}$ reported that E coli was sensitive to cotrimoxazole (80 \%), ciprofloxacin $(91.3 \%)$, nitrofurantoin $(98.2 \%)$.

Current study showed that the most common antibiotics to which Escherichia coli were resistant were amoxicillin, ampicillin, cefazolin, cefoxitin, cefepime, ceftazidime, meropenem, ceftriaxone, sulfamethoxazole and nitrofurantoin by different percent. Arredondo and Amabile (16) found more than $70 \%$ of E coli were resistant to AMP and 32.6 $\%$ to ciprofloxacin. However, another study showed that $\mathrm{E}$ coli were resistant to amoxycillin-clavulanic acid in $68.6 \%-86 \%$ of cases ${ }^{(17)}$.

Susceptibility pattern of Klebsiella isolated from cultures to antibiotics were amikacin $(83.3 \%)$, ceftazidime $(50 \%)$, followed by gentamycin and meropenem $(33.3 \%)$. The most common antibiotics to which Klebsiella were resistant were amoxicillin, ampicillin, cefepime, cefazolin, cefoxitin. Our results agree with Anandkumar et al. ${ }^{(18)}$ who found that Klebsiella was sensitive to amikacin, azithromycin, ceftazidime, amoxicillin-clavulanic acid in $100 \%$ of cases, to ceftriaxone, ciprofloxacin and nitrofurantoin in $75 \%$ of cases, then to cotrimoxazole and gentamycin in $50 \%$ of children. klebsiella species showed $75.3 \%$ resistance to 3 or more drugs but this disagrees with Anandkumar et al. ${ }^{(\mathbf{1 8})}$ for sensitivity to amoxicillin.

The most sensitive antibiotics to Staph aureus were amoxicillin-clavulanate, ampicillin, erythromycin, vancomycin, rifampicin, nitrofurantoin and linezolid. The most resistant

This article is an open access article distributed under the terms and conditions of the Creative Commons Attribution (CC BY-SA) license (http://creativecommons.org/licenses/by/4.0/) 
antibiotic to Staph aureus were cefoxitin, ciprofloxacin and gentamycin. However, Dash et al. ${ }^{(19)}$ found that the sensitivity of Staphylococcus aureus organism to ceftriaxone, amikacin, piperacillintazobactam and vancomycin was very promising out of which vancomycin showed $100 \%$ coverage. Their sensitivities to commonly used antibiotics such as cefixime and ampicillin were low.

Finally, there is an increasing trend of resistance by common bacteria to routine antibiotics. This had been noted in the region and thus the scientific management of UTI needs a continuous updating depending on the antimicrobial sensitivity of the area's or country's key uropathogens.

\section{CONCLUSION}

Identifying the bacterial causes of UTI and their susceptibility pattern will help in selecting the empirical antibiotic therapy till the results of culture are awaited. E. coli is the commonest organism causing UTI followed by klebsiella and proteus mirabilis in children in our study. The isolated organisms showed great resistance to parenteral and oral antibiotics. Amikacin gentamycin may be the first option of empiric therapy while waiting for culture reports. Ceftazidime and ciprofloxacin could be a reserve. Although dipstick and microscopic urinalysis can support the diagnosis of UTI, growth of a single type of bacteria on urine culture is the gold standard.

\section{RECOMMENDATIONS}

It should be asked for urine analysis and culture for any nephrotic syndrome patients either symptomatic or asymptomatic to avoid prolonged hospital stay or relapse thereby to prevent long term renal damage. Constant updating for antimicrobial susceptibility of the main uropathogens of the area should be done. Antibiotic stewardship program is highly encouraged to prevent the hazardous consequences of antibiotic resistance.

\section{REFERENCES}

1. Kamei K, Takahashi M, Fuyama M et al. (2015): Rituximab-associated agranulocytosis in children with refractory idiopathic nephrotic syndrome: case series and review of literature. Nephrol Dial Transpl., 30 (1): 91-96.

2. Hahn D, Hodson E, Willis $\mathbf{N}$ et al. (2015): Corticosteroid therapy for nephrotic syndrome in children. Cochrane Database Syst Rev., 18 (3): CD001533.

3. Sinha A, Bajpai J, Saini S et al. (2014): Serum-soluble urokinase receptor levels do not distinguish focal segmental glomerulosclerosis from other causes of nephrotic syndrome in children. Kidney Int., 85: 649-58.

4. Sreenivasa B, Murthy C, Raghavendra K (2015): Urinary tract infection at presentation of nephrotic syndrome: a clinical evaluation. IJCH., 2: 1-4.

5. Morris B, Wiswell T (2013): Circumcision and lifetime risk of urinary tract infection: a systematic review and metaanalysis. J Urol., 189 (6): 2118-124.

6. Stein R, Dogan H, Hoebeke $P$ et al. (2015): Urinary tract infections in children: EAU / ESPU guidelines. Eur Urol Suppl., 67 (3): 546-58.

7. Centers for Disease Control and Prevention (CDC) (2016): Definition of Nosocomial infections. Infect control J., 7 (3): 40-48.

8. Magiorakos A, Srinivasan A, Carey $\mathrm{R}$ et al. (2012): Multidrug-resistant, extensively drug-resistant and pandrugresistant bacteria: an international expert proposal for interim standard definitions for acquired resistance. Clin Microbiol Infect., 18 (3): 268-281.

9. Ajayan P, Krishnamurthy S, Biswal N et al. (2013): Clinical spectrum and predictive risk factors of major infections in hospitalized children with nephrotic syndrome. Indian Pediatr., 50 (8): 779-781.

10. Robinson J, Finlay J, Lang $M$ et al. (2014): Urinary tract infections in infants and children: Diagnosis and management. Pediatric Child Health, 19 (6): 315-9.

11. Nimri L, Sulaiman M, Hani O (2017): Community-acquired urinary tract infections caused by Burkholderia cepacia complex in patients with no underlying risk factor. JMM Case Rep., 4 (1): e005081.

12. Edlin R, Shapiro D, Hersh A et al. (2013): Antibiotic resistance patterns of outpatient pediatric urinary tract infections. J Urol., 190:222-227.

13. Paschke A, Zaoutis T, Conway $P$ et al. (2010): Previous antimicrobial exposure is associated with drug-resistant urinary tract infections in children. Pediatrics, 125: 664-672.

14. Ronald A (2003): The etiology of urinary tract infection: traditional and emerging pathogens. Dis Mon., 49: 71-82.

15. Mazzalli T (2002): Resistance Trends in Urinary Tract Pathogens and Impact on Management. J Urol., 168: 17201722.

16. Arredondo L, Amabile C (2008): High Resistance Prevalence towards Ampicillin, Cotrimoxasole and Ciprofloxacillin among Uropathogenic $\mathrm{E}$ coli Isolates in Mexico City. J Infect Dev Citres., 2: 350-353.

17. Barua T, Sen S, Sharma J et al. (2017): Pattern of Antimicrobial Sensitivity of Organisms Causing Urinary Tract Infection in Nephrotic Syndrome in Children. Bangladesh J Chil Heal., 40 (3): 154-159.

18. Anandkumar H, Kapur I, Dayan A (2003): Increasing Prevalence of Antibiotic Resistance and Multidrug Resistance among Uropathogens. J Communn Dis., 35: 102-108.

19. Dash D, Bisoi S, Mohanty $M$ et al. (2017): To study the frequency, etiology and predisposing factors of urinary tract infection in children with nephrotic syndrome in Eastern Odisha Region: a hospital based study. Int J Contemp Pediatr., 4 (1): 140-144. 